TITLE:

\title{
Ultrafast Carrier Dynamics in Graphene under a High Electric Field
}

\section{$\operatorname{AUTHOR}(S)$ :}

Tani, Shuntaro; Blanchard, François; Tanaka, Koichiro

\section{CITATION:}

Tani, Shuntaro ... [et al]. Ultrafast Carrier Dynamics in Graphene under a High Electric Field. Physical Review Letters 2012, 109(16): 166603.

ISSUE DATE:

2012-10

URL:

http://hdl.handle.net/2433/162732

RIGHT:

(C) 2012 American Physical Society 


\title{
Ultrafast Carrier Dynamics in Graphene under a High Electric Field
}

\author{
Shuntaro Tani, ${ }^{1,2, *}$ François Blanchard, ${ }^{2,3}$ and Koichiro Tanaka ${ }^{1,2,3, \dagger}$ \\ ${ }^{1}$ Department of Physics, Kyoto University, Sakyo-ku, Kyoto 606-8502, Japan \\ ${ }^{2}$ CREST, Japan Science and Technology Agency, Kawaguchi, Saitama 332-0012, Japan \\ ${ }^{3}$ Institute for Integrated Cell-Material Sciences (WPI-iCeMS), Kyoto University, Sakyo-ku, Kyoto 606-8501, Japan
}

(Received 1 June 2012; published 17 October 2012)

\begin{abstract}
We investigated ultrafast carrier dynamics in graphene with near-infrared transient absorption measurement after intense half-cycle terahertz pulse excitation. The terahertz electric field efficiently drives the carriers, inducing large transparency in the near-infrared region. Theoretical calculations using the Boltzmann transport equation quantitatively reproduce the experimental findings. This good agreement suggests that the intense terahertz field should promote a remarkable impact ionization process and increase the carrier density.
\end{abstract}

Graphene possesses many unique properties arising from its truly 2D honeycomb structure, and offers us a solid state playground of Dirac fermions [1]. One exciting property in graphene is its extraordinary transport property owing to its massless band structure and high Fermi velocity [2], which is significant for both fundamental physics and industrial applications. Although great achievements of dc electron transport in graphene [2-6], current saturation restricts electron transport under a high electric field with field strength over $10 \mathrm{kV} / \mathrm{cm}$, and has been attributed to optical phonon emission in high-energy states of Dirac electrons [5-8]. For anticipated graphene based nanoelectronics devices, the phonon scattering may restrict high field operations.

Thanks to recent advances in high-power and timeresolved terahertz (THz) technologies [9], we can apply electric fields of high strength $(\mathrm{MV} / \mathrm{cm})$ with a pulse width of $\sim 100$ fs to probe the ultrafast nonlinear optical properties of materials in the $\mathrm{THz}$ frequency region. For instance, novel carrier dynamics in semiconductors have been reported such as THz-field-driven mass anisotropy in doped InGaAs [10], and highly efficient carrier multiplication in GaAs quantum wells [11], to name only two. In graphene, S. Winnerl et al. have performed THz-pump THz-probe measurements, revealing that the excitation and relaxation dynamics of carriers strongly depend on the excitation frequency in the $\mathrm{THz}$ region [12]. In these studies, $\mathrm{THz}$ pulses are of picosecond duration, which is longer than the typical optical phonon emission time scale in graphene. A shorter pulse is required to prevail over current saturation caused by optical phonon emission.

In this Letter, we investigated ultrafast carrier dynamics under high electric field in graphene using $200 \mathrm{fs}$ half-cycle $\mathrm{THz}$ excitation pulses and monitoring their dynamics with 50 fs long near-infrared (NIR) probe pulses. Large THz induced transparency is observed in the NIR region for the first time. Numerical calculations using the Boltzmann equation quantitatively reproduce the experimental findings and demonstrate the importance of efficient carrier multiplication by impact ionization in graphene. This multiplication achieved by ultrashort $\mathrm{THz}$ pulse excitation may drastically suppress the current saturation effect.

The sample used in our experiment is a commercial CVD graphene grown on $\mathrm{Cu}$ foil, which is transferred onto a $\mathrm{SiO}_{2}$ substrate. We have confirmed the sample's single-layer nature and uniformity by confocal Raman imaging microscope [13]. The transmissivity of our sample at $800 \mathrm{~nm}$ is $97 \%$, comparable with the value obtained from universal conductivity of intrinsic single layer graphene [1]. Because graphene is deposited on $\mathrm{SiO}_{2}$, substrateinduced inhomogeneity at the graphene-oxide interface gives rise to $p$-type doping with Fermi energy over $200 \mathrm{meV}$ [14].

To investigate hot electron transport properties in graphene, we performed transient absorption measurement in the NIR region under intense $\mathrm{THz}$ excitation, as illustrated in Fig. 1(a). We used a collinear air plasma method for generation of intense half-cycle THz pulses [15] [Fig. 1(b)]. An $800 \mathrm{~nm}$ laser pulse (Ti:Sapphire amplifier that delivers $3 \mathrm{~mJ}$ with $35 \mathrm{fs}$ duration laser pulses) was split into two pulses for the $\mathrm{THz}$ generation $(2.4 \mathrm{~mJ})$ and the timeresolved transmission probe $(<1 \mathrm{~nJ})$. We used a collinear air plasma method for generation of intense half-cycle $\mathrm{THz}$ pulses. An undoped germanium substrate was inserted in the $\mathrm{THz}$ excitation line to remove any remaining $800 \mathrm{~nm}$ pump beam after $\mathrm{THz}$ generation. The $\mathrm{THz}$ pulse was focused on the sample by an off axis parabolic mirror. With some time delay, the $800 \mathrm{~nm}$ probe pulse was also focused at the same spot on the sample. The spot size of the probe pulse $(30 \mu \mathrm{m})$ was carefully taken smaller than that of the THz excitation pulse $(80 \mu \mathrm{m})$ to ensure a homogeneous excitation. The probe light intensity after passing through the sample was measured with an amplified photodiode. A lock-in amplifier (SRS SR830), connected to the photodiode and referenced to the chopper that switched the excitation on and off at $500 \mathrm{~Hz}$, was used to detect the probe 


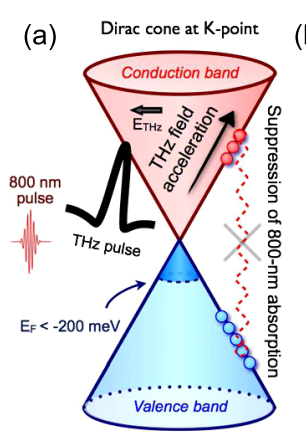

(b)
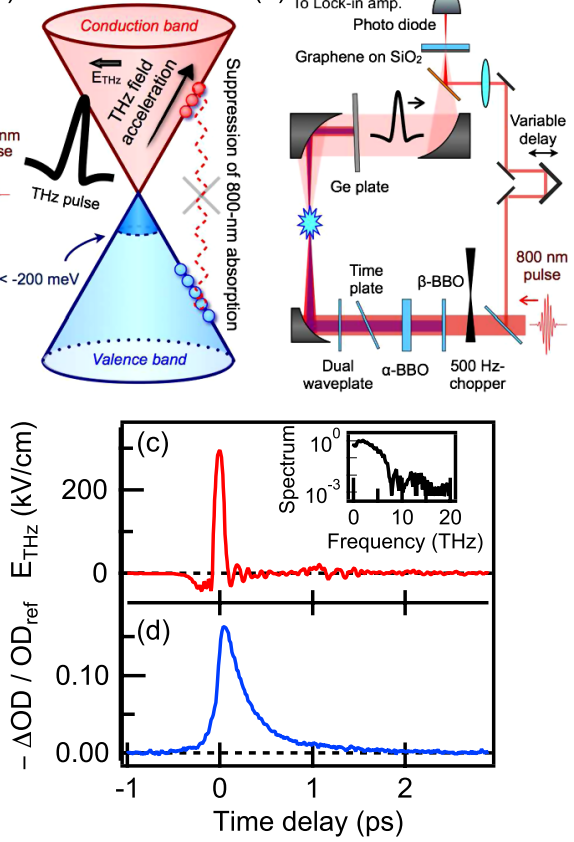

FIG. 1 (color online). (a) Schematic figure of THz-pump NIR-transient absorption measurement in a Dirac cone. (b) Experimental setup. An $800 \mathrm{~nm}$ laser pulse was frequency doubled using $\beta$-barium borate crystal. The relative phase between fundamental and SHG pulses was tuned using an $\alpha$-barium borate crystal and a time plate to maximize the overlap of the two pulses at the focus position. A generated $\mathrm{THz}$ pulse was focused on the sample, which was replaced with a thin GaP crystal when measuring the $\mathrm{THz}$ electric field. (c) Incident $\mathrm{THz}$ electric field and its spectrum. (d) $\mathrm{THz}$ induced normalized differential optical density of graphene at $800 \mathrm{~nm}$ as a function of delay time.

light intensity change by the $\mathrm{THz}$ excitation. The temporal waveform of the $\mathrm{THz}$ pulse was measured using an electro-optical sampling technique by replacing the sample with a $50 \mu \mathrm{m}$ GaP crystal, in which THz-fieldinduced birefringence rotates the polarization of the probe pulse proportional to the field strength. Figure 1(c) shows a temporal profile of the $\mathrm{THz}$ pulse with a peak electric field of $300 \mathrm{kV} / \mathrm{cm}$ and a full-width at half-maximum of $\sim 100 \mathrm{fs}$. Notice that the spectrum of the $\mathrm{THz}$ pulse covers from 0.5 to $7 \mathrm{THz}$ [inset of Fig. 1(c)] with the photon energy several tens of times smaller than that of the probe pulse. All measurements were performed at room temperature and under dehydrated air to suppress $\mathrm{THz}$ absorption from water vapors.

Figure 1(d) shows a typical $\mathrm{THz}$ induced differential optical density (OD) measurement as a function of time with a peak $\mathrm{THz}$ electric field of $300 \mathrm{kV} / \mathrm{cm}$. To clarify the physical meaning observed here, we define the normalized differential OD by $\Delta \mathrm{OD} / \mathrm{OD}_{\text {ref }}=\left(\mathrm{OD}_{\mathrm{ex}}-\mathrm{OD}_{\text {ref }}\right) /$ $\mathrm{OD}_{\text {ref }} \simeq \Delta \mathrm{T} / \mathrm{OD}_{\text {ref }}$, where $\mathrm{OD}_{\text {ref }}$ is the OD of graphene at $800 \mathrm{~nm}$ without $\mathrm{THz}$ pulse excitation, and $\mathrm{OD}_{\mathrm{ex}}$ is that with $\mathrm{THz}$ pulse excitation. The value of $-\Delta \mathrm{OD} / \mathrm{OD}_{\text {ref }}$ corresponds to the population occupation at the energy of an $800 \mathrm{~nm}$ transition. After $\mathrm{THz}$ pulse excitation, the differential OD immediately goes negative (e.g., graphene becomes transparent in the NIR region under THz pulse excitation) and returns to zero within 2 ps. Experimentally, the maximum value of $\Delta \mathrm{T}$ reaches $5 \times 10^{-3}$ for a peak electric field of $300 \mathrm{kV} / \mathrm{cm}$. This large induced transparency over $16 \%$ should be caused by electron filling or hole depletion of the corresponding energy levels.

Figures 2(a) and 2(b) show the temporal profile of induced NIR transparency for peak electric fields of 170 and $300 \mathrm{kV} / \mathrm{cm}$. The maximum values of induced transparency drastically increase for the higher electric field, while carrier relaxation time tends to increase linearly with field strength. The origins leading to the nonlinearity will be discussed in subsequent paragraphs.

Figure 2(e) shows the maximum values of induced transparency as a function of a peak electric field. A power law with exponent 2.5 is found experimentally. This is in contrast with ultrafast optical pump-probe experiments $[16,17]$, which reported a quadratic dependence on the electric field at low fluences and saturation for higher fluences. With optical excitation, the field dependence cannot exceed a power law with exponent 2 because of a limited number of interband transition dipoles $[18,19]$. The superquadratic dependence found here suggests nonlinear coupling between the $\mathrm{THz}$ field and electronic system, and can be explained by the rapid increase in the number of carriers with field strength.

As examined in detail in previous studies from $\mathrm{THz}$ to NIR regions [12,20,21], electromagnetic wave excitation
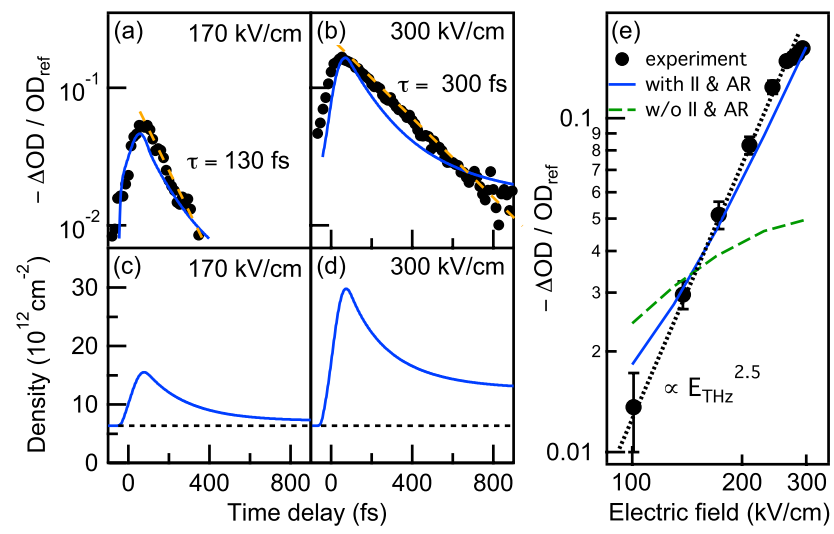

FIG. 2 (color online). THz induced normalized differential optical density as a function of time delay with peak electric fields of (a) $170 \mathrm{kV} / \mathrm{cm}$ and (b) $300 \mathrm{kV} / \mathrm{cm}$ : experiment (circle) and simulation (solid line). The relaxation time $\tau$ is obtained by a single exponential fit (dashed line). (c), (d) Calculated carrier density as a function of time delay. Dashed lines represent initial carrier density. (e) $\mathrm{THz}$ induced transparency of graphene as a function of a peak electric field: experiment (filled circle) and simulations (solid and dashed lines). Experimental results follow a power law with exponent 2.5 (dotted line). 
results in two possible scenarios: interband and intraband transitions. Interband transition creates electron-hole pairs with kinetic energy of the incident photon energy, whereas intraband transition induces athermal distribution of carriers in the momentum space. According to these studies, the dominant mechanism should be determined by photon energy and Fermi energy of graphene. In our experimental conditions, the dominant mechanism is restricted to intraband transition because of large negative Fermi energy below $-200 \mathrm{meV}$ coming from the $\mathrm{SiO}_{2}$ substrate. Since the excited carriers have the kinetic energy corresponding to NIR excitation, their relaxation dynamics can be compared with photoexcitation studies such as ultrafast saturation absorption [22], single-color and two-color pump-probe measurement [16,17,23-25], and ultrafast photoluminescence measurement [26], where 8 fs carriercarrier (CC) scattering, 50-120 fs carrier thermalization, $0.1-1$ ps carrier cooling, and 3-15 ps carrier recombination have been reported.

To understand the carrier dynamics based on our observations, we have calculated the distribution function of carriers in $\boldsymbol{k}$ space using the semiclassical Boltzmann equation. The use of the semiclassical Boltzmann equation should be appropriate because the kinetic energy of carriers reaches several hundreds $\mathrm{meV}$ and much larger than the $\mathrm{THz}$ photon energy, so that interband polarization can be ignored. The Boltzmann equation is as follows:

$$
\begin{aligned}
\frac{\partial}{\partial t} f_{\lambda}(\boldsymbol{k}, t)= & -\frac{q_{\lambda}}{\hbar} \boldsymbol{E}(t) \frac{\partial}{\partial \boldsymbol{k}} f_{\lambda}(\boldsymbol{k}, t) \\
& +\int d \boldsymbol{k}^{\prime}\left\{S_{\boldsymbol{k}, \boldsymbol{k}^{\prime}}^{\lambda-\lambda}+S_{\boldsymbol{k}, \boldsymbol{k}^{\prime}}^{\lambda-p h}-S_{\boldsymbol{k}^{\prime}, \boldsymbol{k}}^{\lambda-\lambda}-S_{\boldsymbol{k}^{\prime}, \boldsymbol{k}}^{\lambda-\mathrm{ph}}\right\} \\
& +\int d \boldsymbol{k}^{\prime}\left\{S_{\boldsymbol{k}, \boldsymbol{k}^{\prime}}^{\mathrm{II}, \lambda}+S_{\boldsymbol{k}, \boldsymbol{k}^{\prime}}^{\mathrm{AR}, \lambda}-S_{\boldsymbol{k}^{\prime}, \boldsymbol{k}}^{\mathrm{III} \lambda}-S_{\boldsymbol{k}^{\prime}, \boldsymbol{k}, \lambda}^{\mathrm{AR}, \lambda},\right.
\end{aligned}
$$

where $f_{\lambda}(\boldsymbol{k}, t)$ is a distribution function of electrons and holes $(\lambda=e, h), q_{\lambda}$ is the charge of carrier, and $\boldsymbol{E}(t)$ is the incident $\mathrm{THz}$ electric field on graphene at time $t . S_{\boldsymbol{k}, \boldsymbol{k}^{\prime}}^{\lambda-\lambda}$ represents intraband $\mathrm{CC}$ scattering rates with coulomb interactions [27]. Intervalley $\boldsymbol{k}-\boldsymbol{k}^{\prime}$ scattering is neglected for large momentum exchange [28]. $S_{\boldsymbol{k}, \boldsymbol{k}^{\prime}}^{\lambda-\text { ph }}$ represents intraand interband phonon scattering rate with $\Gamma$ and $K$ optical phonon modes [28]. Electron-phonon coupling strength is from Ref. [29]. $S_{\boldsymbol{k}, \boldsymbol{k}^{\prime}}^{\mathrm{II}, \lambda}$ and $S_{\boldsymbol{k}^{\prime}, \boldsymbol{k}}^{\mathrm{AR}, \lambda}$ represent impact ionization (II) and Auger recombination (AR) rates [30], respectively. The last two terms (II and AR) change the number of carriers through interband $\mathrm{CC}$ scattering as in Fig. 3(1). The II process creates an extra electron-hole pair while losing the kinetic energy of another electron or hole, as schematically shown in Fig. 3. The AR is the inverse process of II, which reduces the number of carriers. The first term represents carrier acceleration on linear dispersion relation $E=\hbar v_{F}|\boldsymbol{k}|$ (Dirac cone) with an electric field, where $E$ is the energy of the carrier and $v_{F}$ is the Fermi energy in graphene. In the second term, momentumresolved hot phonon effects are included [28]. Acoustic phonon scattering negligibly contributes in our observation time scale and was not included in our calculations [12,28].

The occurrence of II and AR in graphene is controversial because of restrictions from energy and momentum conservation laws in the Dirac cone. Although some authors have stated that these processes are prohibited because of a limited number of possible initial and final momentum configurations [31], some experimental and theoretical studies have indicated the possibility of using these processes $[12,24,25,27,30,32]$. Here, we allow these processes by loosening the momentum conservation law by $\Delta k=$ $2 \times 10^{4} \mathrm{~cm}^{-1}(\sim 1 \mathrm{meV}$ in kinetic energy). This may come from imperfection of our CVD graphene, which possibly results in larger II and AR processes [24,33].

Calculated induced NIR transparency is plotted as a function of a peak electric field in Fig. 2(e) (solid line). The experimental results are quantitatively reproduced
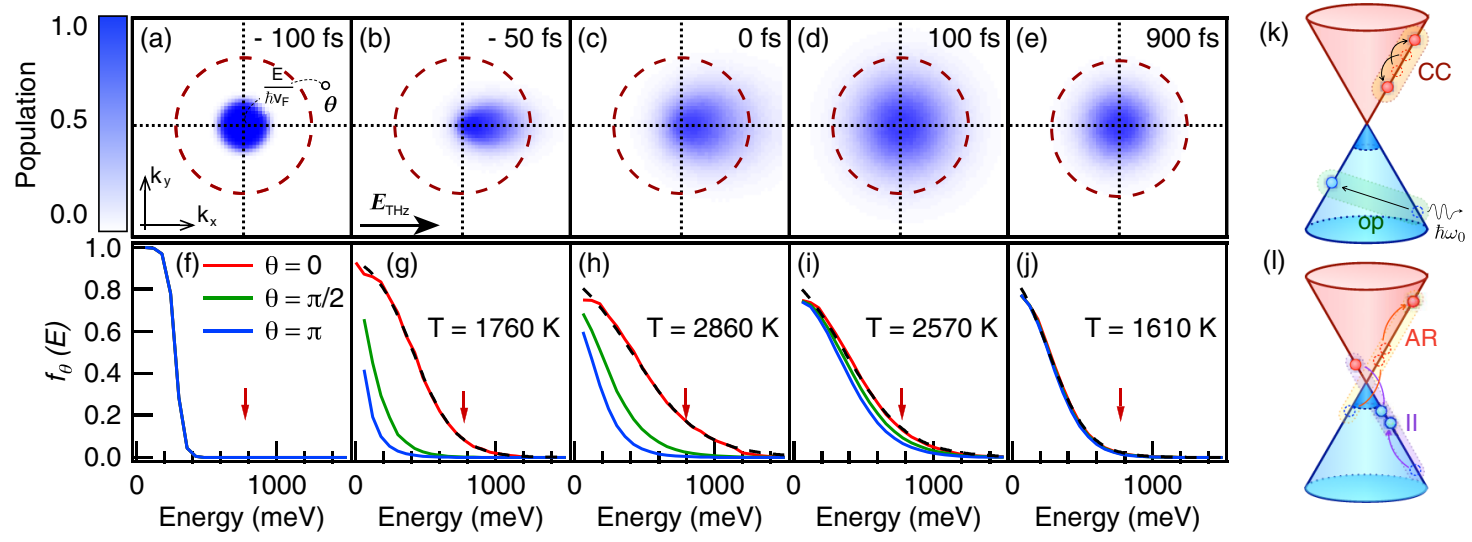

FIG. 3 (color online). (a)-(e) Distribution function of holes in $\boldsymbol{k}$ space with various time delays. Dashed circles represent momenta corresponding to the energy of $800 \mathrm{~nm}$ transition. (f)-(j) Angle-resolved energy distribution of holes. Fermi-Dirac fitting is represented as a dashed line. Vertical arrows indicate the energy of $800 \mathrm{~nm}$ transition. (k), (l) Carrier scattering processes included in the calculation: carrier-carrier scattering (CC), optical phonon scattering (op), impact ionization (II) and Auger recombination (AR). 
when II and AR processes are included. For comparison, the calculations by strictly applying conservation laws (without II and AR) are also plotted as a dashed line. In the latter case, the sublinear scaling with field strength clearly deviates from the experiment for the higher electric field because of rapid optical phonon scattering. We have to stress here that the only adjustable parameter is the initial Fermi energy, which is tuned to reproduce the magnitude of induced transparency in Fig. 2(e). The bestfitted Fermi energy is $-280 \mathrm{meV}$, which is a reasonable value for graphene on $\mathrm{SiO}_{2}$ substrate [14]. Note that the changes of the initial Fermi energy only offset the field dependence without affecting the power law. This good agreement indicated that efficient carrier multiplication by II prevails over AR under the high electric field condition.

To emphasize the effect of carrier multiplication, we plot the calculated carrier density as a function of time in Figs. 2(c) and 2(d). The number of carriers becomes almost five times larger than the initial number of carriers with a peak electric field of $300 \mathrm{kV} / \mathrm{cm}$, resulting in the observed large induced transparency. Recovery of the absorption change at $800 \mathrm{~nm}$ is also calculated and shown as solid lines in Figs. 2(a) and 2(b). Our calculations qualitatively reproduce the field dependent relaxation time, which confirm the longer relaxation time at higher excitation density. This is due to the hot phonon effect [21,28].

Figures 3(a)-3(e) show calculated distribution of holes in $\mathbf{k}$ space at various time delays for the maximum applied electric field of $300 \mathrm{kV} / \mathrm{cm}$. Figures 3(f)-3(h) and 3(j) show the corresponding angle-resolved energy distribution of holes defined as $f_{\theta}(E)=\left\langle f_{h}(E, \phi)\right\rangle_{\theta-\pi / 4<\phi<\theta+\pi / 4}$ with the angle $\theta$ in Fig. 3(a). Before THz pulse excitation, holes are distributed within Fermi energy with $300 \mathrm{~K}$ thermal fluctuation [Figs. 3(a) and 3(f)]. As a THz electric field is applied, the carrier distribution becomes an asymmetric shape [Figs. 3(b) and 3(g), the electric field is rapidly increasing in the positive direction], and keeps its asymmetry until the peak electric field [Figs. 3(c) and 3(h)], although the asymmetry decreases rapidly by optical phonon scattering. After the $\mathrm{THz}$ excitation pulse is passing through the sample, the distribution immediately recovers its symmetric shape [Figs. 3(d) and 3(i)] and returns back to the initial distribution by emitting optical phonons [Figs. 3(e) and 3(j)]. This argument is well confirmed by angle-resolved energy distributions fitted with Fermi-Dirac distribution in the THz field direction $k_{x}$ [Figs. 3(f)-3(j)]. For instance, temperature in the $k_{x}$ direction is $300 \mathrm{~K}$ before excitation and is increased to $2860 \mathrm{~K}$ after $\mathrm{THz}$ pulse excitation, though temperature in the other direction remains low even at the peak electric field [Fig. 3(h)]. After $\mathrm{THz}$ pulse excitation, energy distributions immediately thermalize in all directions and return back to the initial distribution through phonon emission. Notice that the dashed circles in Figs. 3(a)-3(e) represent momenta corresponding to the energy of an $800 \mathrm{~nm}$ transition, also indicated with arrows in Figs. 3(f)-3(j).

In conclusion, we reported the first observation of large THz-induced near-infrared transparency in graphene. Our results were compared with simulations using the Boltzmann equation and we proved that carrier multiplication occurs in graphene driven by high field and ultrashort $\mathrm{THz}$ pulses. The observed dependence of the rise in charge density on $\mathrm{THz}$ peak field strength indicates an interband impact ionization mechanism in graphene. The impact ionization process may suppress optical phonon emission by reducing kinetic energies of carriers while conserving current, which could promote efficient carrier transport suppressing current saturation.

We thank G. Asai for the preparation technique of our sample. This work was supported by Grant-in-Aid for Scientific Research on Innovative Area 'Optical science of dynamically correlated electrons (DYCE)' (Grant No. 20104007), Grant-in-Aid for Scientific Research (A) (Grant No. 23244065), and Grant-in-Aid for the Global COE Program 'The Next Generation of Physics, Spun from Universality and Emergence' from the MEXT of Japan.

*tani@scphys.kyoto-u.ac.jp

${ }^{\dagger}$ kochan@scphys.kyoto-u.ac.jp

[1] K. Novoselov, A. Geim, S. Morozov, D. Jiang, M. Grigorieva, S. Dubonos, and A. Firsov, Nature (London) 438, 197 (2005).

[2] J.-H. Chen, C. Jang, S. Xiao, M. Ishigami, and M. S. Fuhrer, Nature Nanotech. 3, 206 (2008).

[3] S. D. Sarma, S. Adam, E. Hwang, and E. Rossi, Rev. Mod. Phys. 83, 407 (2011).

[4] X. Du, I. Skachko, A. Barker, and E. Y. Andrei, Nature Nanotech. 3, 491 (2008).

[5] S. Berciaud, M. Y. Han, K. F. Mak, L. E. Brus, P. Kim, and T.F. Heinz, Phys. Rev. Lett. 104, 227401 (2010).

[6] I. Meric, M. Y. Han, A. F. Young, B. Ozyilmaz, P. Kim, and K. L. Shepard, Nature Nanotech. 3, 654 (2008).

[7] A. Barreiro, M. Lazzeri, J. Moser, F. Mauri, and A. Bachtold, Phys. Rev. Lett. 103, 076601 (2009).

[8] W. Tse, E. Hwang, and S. Sarma, Appl. Phys. Lett. 93, 023128 (2008).

[9] M. C. Hoffmann and J. A. Fülöp, J. Phys. D 44, 083001 (2011).

[10] F. Blanchard, D. Golde, F. H. Su, L. Razzari, G. Sharma, R. Morandotti, T. Ozaki, M. Reid, M. Kira, S. W. Koch, and F. A. Hegmann, Phys. Rev. Lett. 107, 107401 (2011).

[11] H. Hirori, K. Shinokita, M. Shirai, S. Tani, Y. Kadoya, and K. Tanaka, Nature Commun. 2, 594 (2011).

[12] S. Winnerl, M. Orlita, P. Plochocka, P. Kossacki, M. Potemski, T. Winzer, E. Malic, A. Knorr, M. Sprinkle, C. Berger, W. A. de Heer, H. Schneider, and M. Helm, Phys. Rev. Lett. 107, 237401 (2011). 
[13] A. C. Ferrari, J. C. Meyer, V. Scardaci, C. Casiraghi, M. Lazzeri, F. Mauri, S. Piscanec, D. Jiang, K. S. Novoselov, S. Roth, and A. K. Geim, Phys. Rev. Lett. 97, 187401 (2006).

[14] S. Ryu, L. Liu, S. Berciaud, Y. Yu, H. Liu, P. Kim, G. Flynn, and L. Brus, Nano Lett. 10, 4944 (2010).

[15] J. Dai, N. Karpowicz, and X. C. Zhang, Phys. Rev. Lett. 103, 023001 (2009).

[16] J. Dawlaty, S. Shivaraman, M. Chandrashekhar, F. Rana, and M. Spencer, Appl. Phys. Lett. 92, 042116 (2008).

[17] F. Carbone, G. Aubock, A. Cannizzo, F. V. Mourik, R. Nair, A. Geim, K. Novoselov, and M. Chergui, Chem. Phys. Lett. 504, 37 (2011).

[18] T. Li, L. Luo, M. Hupalo, J. Zhang, M. C. Tringides, J. Schmalian, and J. Wang, Phys. Rev. Lett. 108, 167401 (2012).

[19] Z. Sun, T. Hasan, F. Torrisi, D. Popa, G. Privitera, F. Wang, F. Bonaccorso, D. M. Basko, and A.C. Ferrari, ACS Nano 4, 803 (2010).

[20] K. F. Mak, M. Y. Sfeir, Y. Wu, C. H. Lui, J. A. Misewich, and T. F. Heinz, Phys. Rev. Lett. 101, 196405 (2008).

[21] D. Sun, Z.-K. Wu, C. Divin, X. Li, C. Berger, W. A. de Heer, P. N. First, and T. B. Norris, Phys. Rev. Lett. 101, 157402 (2008).
[22] G. Xing, H. Guo, X. Zhang, T. Sum, and A. Huan, Opt. Express 18, 4564 (2010).

[23] T. Kampfrath, L. Perfetti, F. Schapper, C. Frischkorn, and M. Wolf, Phys. Rev. Lett. 95, 187403 (2005).

[24] P. George, J. Strait, J. Dawlaty, S. Shivaraman, M. Chandrashekhar, F. Rana, and M. Spencer, Nano Lett. 8, 4248 (2008).

[25] M. Breusing, S. Kuehn, T. Winzer, E. Malić, F. Milde, N. Severin, J. P. Rabe, C. Ropers, A. Knorr, and T. Elsaesser, Phys. Rev. B 83, 153410 (2011).

[26] C. H. Lui, K. F. Mak, J. Shan, and T. F. Heinz, Phys. Rev. Lett. 105, 127404 (2010).

[27] A. Girdhar and J. Leburton, Appl. Phys. Lett. 99, 043107 (2011).

[28] E. Malic, T. Winzer, E. Bobkin, and A. Knorr, Phys. Rev. B 84, 205406 (2011).

[29] S. Piscanec, M. Lazzeri, F. Mauri, A. C. Ferrari, and J. Robertson, Phys. Rev. Lett. 93, 185503 (2004).

[30] F. Rana, Phys. Rev. B 76, 155431 (2007).

[31] M.S. Foster and I. L. Aleiner, Phys. Rev. B 79, 085415 (2009).

[32] T. Winzer, A. Knorr, and E. Malic, Nano Lett. 10, 4839 (2010).

[33] C. Grein and H. Ehrenreich, J. Appl. Phys. 93, 1075 (2003). 